Special Issue: Organogenesis

\title{
Long noncoding RNAs in organogenesis: making the difference
}

\author{
Phillip Grote ${ }^{1}$ and Bernhard G Herrmann ${ }^{2,3}$ \\ ${ }^{1}$ Institute of Cardiovascular Regeneration, Center for Molecular Medicine, Goethe University, Theodor-Stern-Kai 7, 60590, \\ Frankfurt am Main, Germany \\ ${ }^{2}$ Max Planck Institute for Molecular Genetics, Department of Developmental Genetics, Ihnestr. 63-73, 14195, Berlin, Germany \\ ${ }^{3}$ Charité- University Medicine Berlin, Institute for Medical Genetics, Campus Benjamin Franklin, Hindenburgdamm 30, \\ 12203 Berlin, Germany
}

\begin{abstract}
A large proportion of the cellular transcriptome of higher vertebrates consists of non-protein coding transcripts, among them the long noncoding RNAs (IncRNAs). Although IncRNAs are functionally extremely divergent, many ncRNAs have been shown to interact with chromatin modifying complexes and/or with transcriptional regulators. Via such interactions, many IncRNAs are involved in controlling the activity and expression level of target genes, including important regulators of embryonic processes, and thereby fine-tune gene regulatory networks controlling cell fate, lineage balance, and organogenesis. Intriguingly, an increase in organ complexity during evolution parallels a rise in IncRNA abundance. The current data suggest that IncRNAs support the generation of cell diversity and organ complexity during embryogenesis, and thereby have promoted the evolution of more complex organisms.
\end{abstract}

\section{Genetic control of cell differentiation during embryogenesis}

The diversity of cell types and organs of multicellular organisms develops by differentiation of the descendants of a single totipotent cell, the fertilized egg. As all cells of the developing organism contain the same genome, the differentiation process requires that the genomes of cells taking different fates vary with respect to transcriptionally active and inactive regions. This variation in nuclear organization involves modifications of chromatin causing activation or silencing of genes, and is controlled by differentially expressed developmental regulators, such as transcription factors that are organized in gene regulatory networks (GRN). Besides qualitative differences (i.e., cell-type specific expression), quantitative differences such as the expression levels of regulators appears to play an important role in differentiation processes. This is, for instance, reflected by the fact that many important transcription factors display haplo-insufficiency phenotypes. For example, normal heart development requires that both

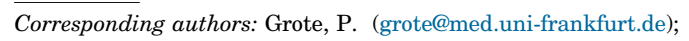

Herrmann, B.G. (herrmann@molgen.mpg.de).

Keywords: lncRNAs; embryo; development; organ; cell lineage.

0168-9525/

(C) 2015 Elsevier Ltd. All rights reserved. http://dx.doi.org/10.1016/j.tig.2015.02.002 alleles of the cardiac transcription factor coding genes Nkx2-5 (NK2 homeobox 5), Gata4 (GATA binding protein 4), and Tbx5 (T-box 5) are functional. If one is dysfunctional or lacking, congenital heart disease lesions develop [1-3]. Hence, the development of a vital organism not only depends on the accurate temporal and spatial control of gene activation, but also, at least for some critical developmental regulators, on appropriate levels of gene expression and activity.

For decades, deciphering the function of individual important transcription factors and chromatin organizing proteins has been the major focus of developmental biologists attempting to understand the control of embryonic processes. Despite these efforts, a comprehensive understanding of the genomic control mechanisms acting in individual cells and cell lineages during embryonic development is still lacking. In this review, we provide an overview of recent studies elucidating the function of lncRNAs in embryonic development and discuss their implications for understanding the role of lncRNAs in fine-tuning GRNs during development, cell diversification, and the evolution of higher organ complexity.

\section{ncRNAs as modulators of gene activity}

Systematic transcriptome analyses by deep sequencing of human cell lines have revealed that close to $75 \%$ of the human genome is transcribed into primary transcripts, but less than $3 \%$ of the genome accounts for protein coding transcripts [4]. Thus, the vast majority of the mammalian genome encodes non-protein coding RNAs (ncRNAs). Analogous to the functional diversity of proteins, ncRNA transcripts are highly heterogeneous with respect to size and function. Some well investigated classes of ncRNAs, including rRNAs, small nucleolar RNAs (snoRNAs), and tRNAs, have been shown to play important roles in mRNA translation or splicing. Furthermore, short RNA molecules of 21-28 nucleotides in length, such as miRNAs, siRNAs, and PIWI-interacting RNAs (piRNAs), are involved in transcriptional or post-transcriptional RNA silencing, and thus in fine-tuning target gene activity at the transcript level. Finally, numerous circular RNA species (circRNAs) [5] that are expressed in a tissue- and developmental stage-specific manner [6] have recently been discovered and presumably are also involved in the control 
of regulatory networks. Some of these circRNAs may act as sponges for miRNAs, thereby adding an additional regulatory layer [6].

\section{Re-emergence of a neglected ncRNA species: the long noncoding RNAs}

The extraordinary advance in sequencing technology, allowing the detection of low abundance transcripts in genomewide transcriptome analyses via massive parallel sequencing, has put a class of ncRNAs in the spotlight that has been known but neglected for a long time. These so-called long noncoding RNAs (lncRNAs) are by definition longer than 200 nucleotides and have limited or no protein-coding potential. However, many lncRNAs associate with ribosomes and, hence, might have the potential to encode short peptides, but to what extent this actually occurs remains to be investigated $[7,8]$. In addition, some lncRNAs might have dual roles, acting as both full-length transcripts and as precursors of small RNAs [9]. In general, lncRNAs tend to be expressed at a lower level than mRNAs [4]. The RNA stability of lncRNAs was found to be only slightly decreased as compared to mRNAs, suggesting that the lower transcript level is the result of a reduced transcription rate [10].

LncRNA genes exist in various genomic contexts. Like mRNA genes they can have their own promoters and regulatory elements, distant from any known mRNA gene locus. However, many lncRNAs overlap with mRNA transcripts in sense or antisense orientation, or are transcribed from introns. A large subset of lncRNAs is transcribed divergently (antisense) from mRNA coding genes [11], sharing promoter and regulatory elements with the latter, or lie upstream of neighboring mRNA coding genes and are transcribed in sense orientation into the promoter of the neighbor. LncRNAs can also originate from enhancers (eRNAs) as long unspliced or spliced transcripts $[12,13]$. The number of lncRNA transcripts expressed in human and mouse is in the range of tens of thousands, when summed up over all developmental and adult cell types. However, although the number of studies and databases devoted to lncRNAs is increasing, a well-annotated database across species that integrates all the available information is not yet available.

Intriguingly, many lncRNAs display a more restricted and tissue-specific expression pattern than mRNAs [1416]. How this is achieved is currently an open question. One important hint comes from a recent study showing an association of the promoters of tightly controlled lncRNA genes with super-enhancers, which are highly cell-type specific $[17,18]$. The tight control of lncRNAs suggests specific roles in the differentiation and function of particular cell types. Recent reports have provided a number of examples demonstrating a decisive role of several lncRNAs in embryonic development and organogenesis (Table 1), discussed in detail below.

\section{IncRNAs acting in epigenetic control during mammalian development}

Numerous lncRNAs interact with various types of proteins involved in histone modification, chromatin remodeling, or DNA methylation. In particular, the Polycomb Repressive Complex 2 (PRC2), which is essential for embryonic development, binds thousands of ncRNAs, including numerous lncRNAs [19-21]. This finding sparked the idea that lncRNAs might be involved in targeting PRC2 to specific gene control elements.

For instance, Xist (X-inactivation specific transcript) binds to PRC2, thereby causing extensive tri-methylation of histone 3 lysine 27 (H3K27me3) along the X-chromosome, followed by inactivation of the marked copy, a mechanism required for dosage compensation in (mammalian) females [22]. Several other lncRNAs exerting their function through binding to $\mathrm{PRC} 2$ have recently been identified. One well-studied example is Hotair (HOX antisense intergenic RNA), which regulates the expression of genes in the HoxD cluster and multiple imprinted genes [23]. The loss of Hotair in null-mutant mouse embryos results in homeotic transformations and fusions of carpal elements in the wrist [23]. Another example is Fendrr (fetal-lethal noncoding developmental regulatory RNA). Interaction of Fendrr with PRC2 is required to adjust the expression levels of its target genes that are involved in the control of lateral mesoderm development. Remarkably, the two direct target genes of Fendrr identified so far, Foxf1 (Forkhead-box F1) and Pitx2 (paired-like homeodomain transcription factor 2), are transcription factors that play pivotal roles in lateral mesoderm differentiation, and show haplo-insufficiency phenotypes in mutants expressing only one functional allele. The targeting of PRC2 to the promoters of these transcription factors by Fendrr thus plays an important role in fine-tuning the expression levels of these dosage-sensitive regulators. Genetic ablation of the Fendrr transcript results in malformations of the heart and the body wall, which originate from the lateral mesoderm, and eventually in embryonic death $[24,25]$. Another lncRNA important for the specification of the early heart cell lineage is Braveheart, which interacts with the PRC2 component SUZ12 [26]. Braveheart is required for the activation of a network of genes that establish the cardiac lineage from lateral mesoderm progenitors. Interestingly, Braveheart acts upstream of Mesp1, a transcriptional regulator and early pioneering factor of the cardiac cell lineage, which in turn might regulate Fendrr expression at the transcriptional level [27]. Hence, these latter two lncRNAs act together with PRC2 in a regulatory network controlling the early cardiac cell lineage (Figure 1A).

HOTTIP (HOXA transcript at the distal tip) was the first lncRNA shown to interact with WDR5 (WD repeat domain 5) [28]. The inactivation of HOTTIP in human fibroblasts by small hairpin RNAs (shRNAs) reduces the occupancy of MLL1 (mixed lineage leukemia 1) and WDR5 near transcription start sites (TSS) across the HOXA gene cluster, which is particularly prominent in the $5^{\prime}$-region of this gene cluster [28]. This results in lower expression of HOXA target genes of HOTTIP. Virus-assisted inactivation of HOTTIP by shRNAs in chick embryos causes the shortening of distal bony elements in the forelimbs [28]. In a follow-up study, an RNA binding pocket was identified in WDR5, which provides a specific interface for binding of HOTTIP and other lncRNAs [29]. Notably, ES cells expressing a mutant form of WDR5 defective in RNA binding are unable to maintain self-renewal and prematurely undergo differentiation into the ectodermal and mesodermal lineages. This is at least in 
Table 1. Selection of IncRNAs involved in cell lineage and organ development

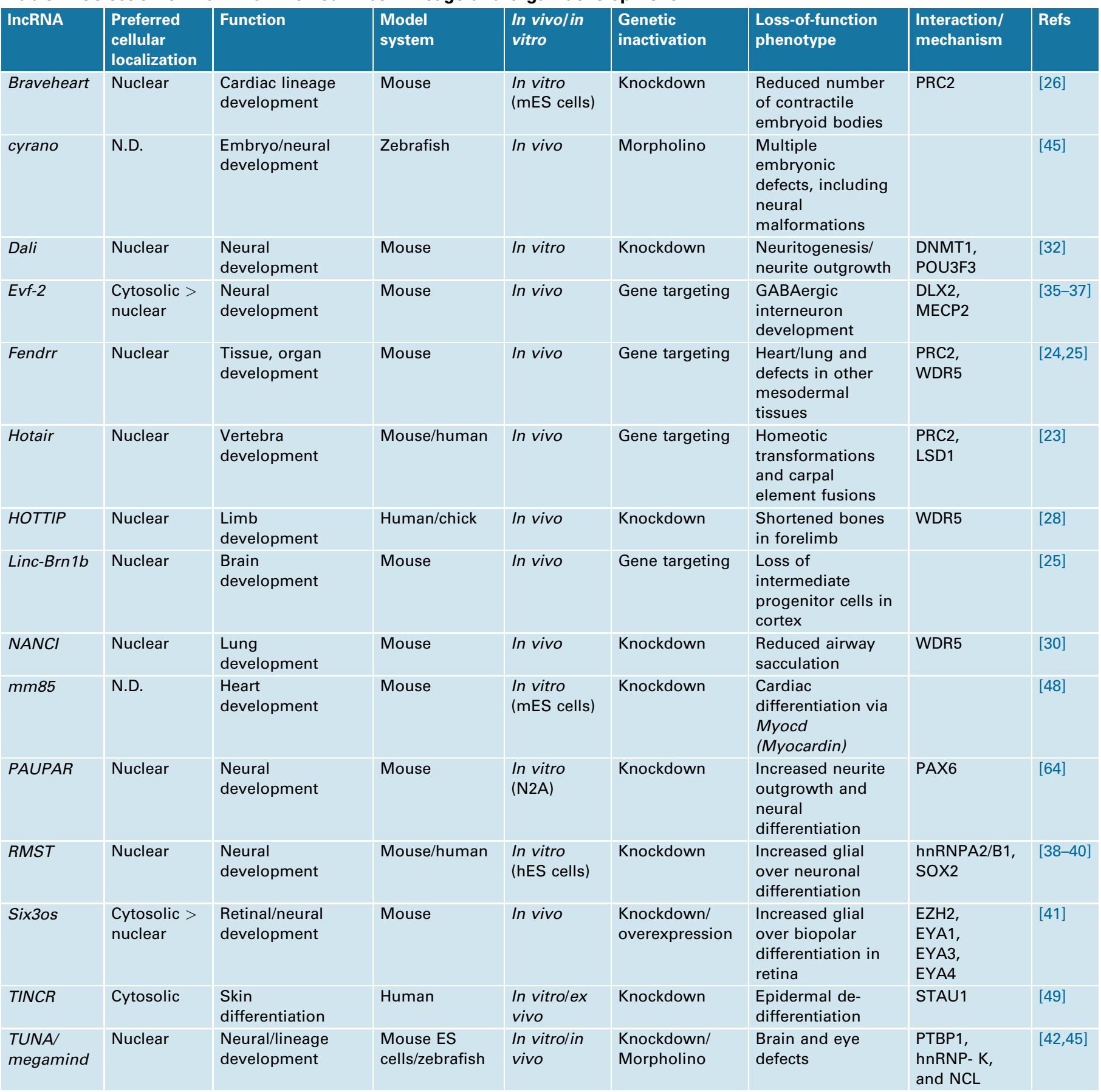

part due to impaired stability of the mutant WDR5 protein [29]. Fendrr interacts with both PRC2 and WDR5, but whether this occurs at the same time is not clear, and Fendrr targets modified via interaction with WDR5 have not been reported [22]. An lncRNA that has been shown to interact with WDR5 and modulate H3K4me3 (histone 3 lysine 4 trimethylation) levels through TrxG/Mll (Trithorax group/ mixed-lineage Leukemia) directly, is NANCI (Nkx2.1associated noncoding intergenic RNA) [30]. It is expressed downstream of and in the same direction as Nkx2.1 (NK2 homeobox 1). The in vivo knockdown of NANCI causes reduced expression of $N k \times 2.1$, coinciding with reduced H3K4me3 levels at its promoter. Nkx2.1 is a transcription factor and important regulator displaying haplo-insufficiency in lung development [30]. NANCI and Nkx2.1 are coexpressed in the developing lung of E12.5 mouse embryos, and depletion of NANCI by shRNA mediated knockdown in vivo phenocopies morphological abnormalities observed in mutant lungs expressing only one functional allele of Nkx2.1.

A number of lncRNAs interact with DNA methylation enzymes and thereby can be involved in imprinting [31]. Recently, Dali (DNMT1-associated long intergenic), which is expressed downstream of the transcription factor gene Pou3f3 (POU domain, class 3, transcription factor 3), has been shown to interact with the DNA methylation maintenance enzyme DNA (cytosine-5)-methyltransferase 


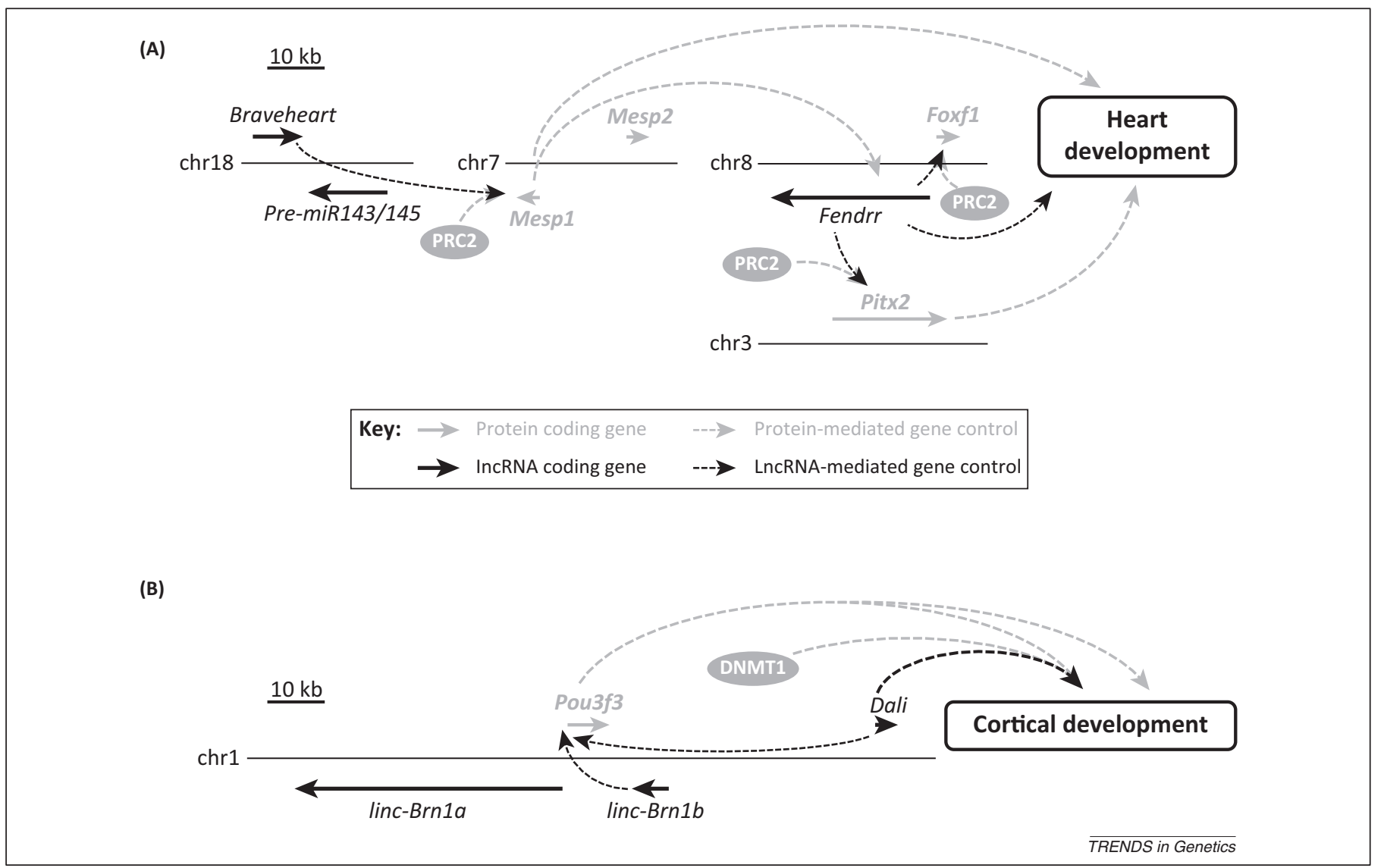

Figure 1. Long noncoding RNAs (IncRNAs) participate in complex gene regulatory networks of organ development. (A) The IncRNAs Braveheart and Fendrr (fetal-lethal noncoding developmental regulatory RNA) interact with Polycomb Repressive Complex 2 (PRC2) to control lateral mesoderm and cardiac transcription factors, together contributing to the gene regulatory network regulating heart development $[24,26,27]$. (B) The IncRNAs linc-Brn1b and Dali regulate the important developmental transcription factor coding gene Pou3f3. Dali interacts with DNA (cytosine-5)-methyltransferase 1 (DNMT1) and with POU3F3 to regulate cortical development [25,32].

1 (DNMT1) and regulate the methylation status of $\mathrm{CpG}$ island-associated promoters. Dali is involved in regulating Pou3f3 transcription. Depletion of Dali during differentiation of neuroblastoma (N2A) cells by RNA knockdown results in fewer cells forming neurites and reduced neurite outgrowth [32]. Furthermore, Dali knockdown results in the dysregulation of key genes of neural development in differentiating cells as compared to control cells, and Dali and POU3F3 share transcriptional targets in this process (Figure 1B). These data established Dali as an important modulator of a GRN acting during neural differentiation.

Several reports have revealed lncRNAs that can interact concurrently with multiple complexes and may serve as scaffolding components for larger complexes. For instance, Hotair interacts with PRC2 and the CoREST/REST/LSD1 complex to coordinate combined targeting of both complexes to genomic regions enabling efficient demethylation of the activating H3K4me2 histone mark and simultaneous setting of the repressive H3K27me3 histone mark [33]. Another example is Kcnq1ot1 (KCNQ1 overlapping transcript 1), which interacts with numerous complexes and complex components, such as PRC2, DNMT1, and G9a (for review see [34]). Similarly, Dali interacts with a number of chromatin modifying complex components, such as BRG1 (Brahma-related gene 1, also: SMARCA4, SWI/SNF related, matrix associated, actin dependent regulator of chromatin, subfamily a, member 4), P66beta (transcriptional repressor p66-beta), and SIN3A (SIN3 transcription regulator family member A) in mice [32]. The identification of common targets of Dali and POU3F3 and the interaction of these factors observed by RNA immunoprecipitation (RIP) suggested that they form a functional complex [32]. Interestingly, mouse mutants carrying a genetic deletion of the entire lncRNA locus linc-Brn1b show significant down-regulation of the Pou3f3 gene and exhibit defects in cortex organization and cortical lamination [25]. This suggests that Dali and linc-Brn1b might act in a gene regulatory network with Pou3f3 to control neuronal development (Figure 1B). In conclusion, at least a subset of lncRNAs presumably forms a bridge between the epigenetic machinery and transcription factors.

\section{IncRNAs as cofactors of transcriptional regulators}

Some lncRNAs have been found to interact with transcription factors important for embryonic development, and thereby act as transcriptional co-regulators. The conserved lncRNA $E v f-2$ (splice variant of Evf-1, embryonic ventral forebrain) cooperates with the transcription factor DLX2 (distal-less homeobox 2) to control the expression of Dlx $5 / 6$ via an enhancer in neuronal (medial ganglionic eminence, MGE) tissue in E13.5 mouse embryos [35]. A stable complex of $E v f-2$ with DLX1/2 proteins binds to the regulatory elements in the Dlx5 / 6 locus in vivo, most likely preventing repressive enhancer DNA-methylation [36,37]. Genetic 
depletion of $E v f-2$ by premature transcriptional termination leads to a reduction of GABAergic interneurons in adult mice due to a decrease in the number of neurons specified for this lineage during embryonic development [37]. Another lncRNA that is specifically expressed in neural tissues, is Rmst (Rhabdomyosarcoma 2-associated transcript) [38]. Rmst interacts with the transcription factor SOX2 (sex determining region Y-box 2) [39], allowing SOX2 to bind to neurogenic promoters and regulate genetic programs required for neural development [40].

Whereas these lncRNAs interact with transcription factors directly, the lncRNA Six3os (SIX homeobox 3 opposite strand) binds to transcriptional co-regulator proteins [EYA1, EYA3, and EYA4 (Eyes absent homolog 1,3,4)] in retinal development [41]. Virus mediated knockdown of Six3os in the developing retina of mouse embryos results in a reduction of bipolar cells and a parallel increase of Muller glial cells in the retina. Thus, Six3os appears to be involved in controlling the balance in the generation of different cell types during development.

Transcription can also be regulated by interaction of lncRNAs with proteins that are not classified as transcriptional regulators. For instance, the lncRNA TUNA/megamind (Tcl1 upstream neuron-associated lincRNA) interacts with several RNA binding proteins [PTBP1 (polypyrimidine tract binding protein 1), hnRNP-K, and NCL/Nucleolin] involved in maintaining the pluripotency of ES cells [4244]. TUNA/RNA binding protein complexes have been detected at the promoters of the pluripotency factors NANOG, SOX2, and fibroblast growth factor 4 (FGF4). TUNA is specifically expressed in ES cells, in the neural lineage, and in the central nervous system of adult mice. TUNA expression increases during neural differentiation of ES cells in vitro, and the depletion of TUNA by RNA knockdown causes a reduction of several neural lineage genes, suggesting an important role in neural development and function. Consistent with these findings obtained in vitro, depletion of TUNA/megamind by morpholino-mediated knockdown in the developing zebrafish embryo results in impaired neural development and impaired locomotor function in touch response tests in zebrafish larvae $[42,45]$.

Transcription factors require the interaction with the transcriptional machinery, in particular the mediator complex, for activating gene transcription. An important subunit of the mediator complex is MED12 (mediator complex subunit 12), which presumably serves as a relay for signals from several important developmental signaling pathways, such as WNT and Hedgehog, which are essential for embryonic development [46]. Interestingly, particular mutations in human MED12 that abrogate the interaction of MED12 with lncRNAs are associated with the human congenital disease Opitz-Kaveggia (FG) syndrome [47].

Furthermore, in the developing lung of mice, the expression of several lncRNAs is correlated with the expression of adjacent transcription factor coding genes [30]. The knockdown of two (NANCI and LL34) lncRNAs identified showed that both play important and distinct roles in endoderm and lung development by controlling the expression of transcription factors critical for the formation of this organ. Similarly, lncRNAs regulate important transcriptional control factors during heart development [48].
Notably, in addition to the above discussed mechanisms, the transcriptome of a cell can be altered by lncRNAs via a plethora of other regulatory functions. An interesting example is the lncRNA TINCR (terminal differentiation-induced ncRNA), which interacts with mRNAs and thereby increases the mRNA abundance of important skin differentiation genes [49]. This outcome is achieved by direct interaction of TINCR with the RNA binding protein STAU1 (double-stranded RNA-binding protein Staufen homolog 1) and, possibly, by stabilization of the target mRNAs.

\section{IncRNAs might promote cell diversification during embryogenesis}

Although only a small number of lncRNAs have been analyzed with respect to their function in embryonic development so far, an interesting common property is emerging from some of these studies. In loss-of-function analyses, the lack of several lncRNAs caused an imbalance in the developmental potential or in the differentiation of particular cell types or lineages, or the dysfunction of organs. This argues that lncRNAs play an important role in controlling cellular fate.

Although the basic body plan of all vertebrates is conserved, different species develop organs with a tremendous range of functional complexity. Increasing organ complexity requires extensive diversification of cell types during embryonic development, which means that during evolution a particular cell type existing in a rather primitive organism diverged into two or several similar but distinct cell types in more complex organisms. However, despite an increase in organ complexity, the core transcription factor networks controlling organogenesis, for instance heart development, are well conserved between, for example, zebrafish and human. Although higher complexity of cellular proteomes via differential splicing and post-translational modifications certainly contributed to the evolution of higher cellular complexity, a crucial factor for evolution of complex organisms appears to be a huge increase in regulatory complexity [50].

The number of lncRNAs rose with increasing organ complexity [51] (Figure 2A), and a considerable fraction of lncRNAs are involved in nuclear organization, chromatin modification, and epigenetic gene control. Thus, lncRNAs are likely to take a considerable role in creating higher complexity in the regulatory mechanisms. This is further supported by the observations that many lncRNAs have a more specific expression pattern than protein coding genes [14,15]. In particular the human brain, the most complex organ of all, expresses a large number of lncRNAs with highly specific expression patterns [52].

It is thus plausible that lncRNAs are involved in finetuning of GRNs controlling the differentiation of specific cell types and lineages, and contribute extensively to the formation and proper function of elaborate tissues and organs from rather simple precursors (Figure 2B) [53]. Moreover, the low sequence conservation of orthologous lncRNAs observed between even closely related species argues that lncRNAs provide a suitable platform for rapid evolution of novel functions.

Adding up the evidence and observations it is conceivable that lncRNAs play an important role in cell diversification 


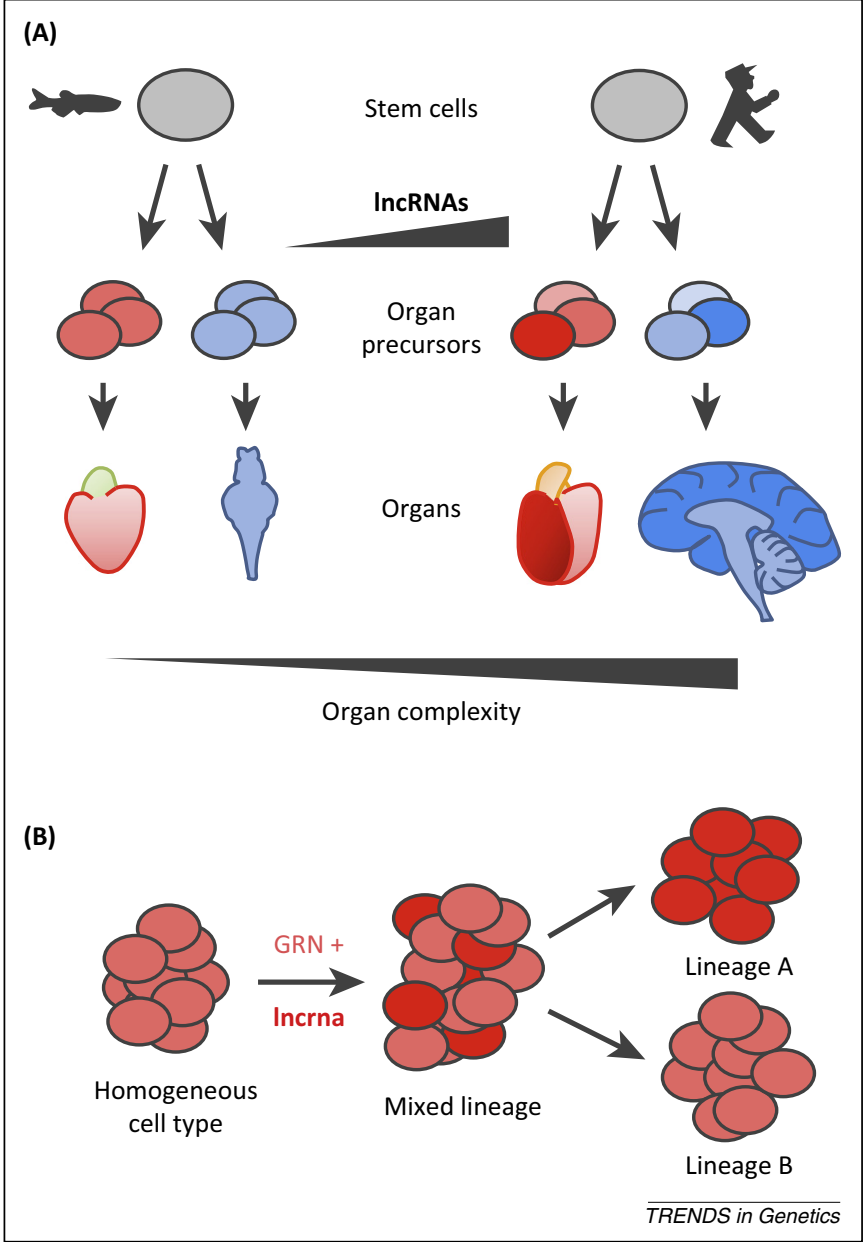

Figure 2. Long noncoding RNAs (IncRNAs) may have contributed to the evolution of higher organ complexity. (A) An increase in organ complexity during evolution is correlated with rising IncRNA gene numbers. Left: single-chambered heart and simple brain in the fish. Right: double-chambered heart and complex brain with enlarged cortex in the human. (B) IncRNAs modulating an evolutionary conserved gene regulatory network (GRN) might promote cell lineage diversification.

during embryogenesis, resulting in more complex tissues and organs during evolution, and thus shaping more sophisticated organisms.

\section{Concluding remarks}

Thorough genetic and molecular investigations of numerous individual lncRNAs will be required to elucidate the full range of mechanisms by which lncRNAs take part in GRNs controlling cell differentiation and diversification.

Careful consideration must be given to the design of genetic manipulations used to interfere with or disrupt lncRNA function, as the type of mutation may have an important impact on the outcome and thus may influence the interpretation of the role of the lncRNA [54]. For example, partially conflicting results have been obtained in studies on MALAT1 (metastasis associated lung adenocarcinoma transcript 1) and lincRNA-p21, depending on whether the genes were inactivated via RNAi or via genetic ablation [55-59]. Furthermore, a recent study in zebrafish, in which antisense RNA (morpholino)-guided mutants (morphants) and genetic mutants of the same proteincoding and noncoding genes were compared, revealed only a poor correlation between the phenotypes [60]. These studies provide strong arguments in favor of genetic inactivation as a more reliable approach.

However, only a small proportion of 18 lncRNA knockout lines generated in mice showed impaired organ development or compromised animal survival [25]. In some instances removal of lncRNAs may have caused an imbalance rather than a dysfunction of the GRNs controlling cell differentiation. It is also likely that some phenotypes may be subtle and only become apparent upon environmental challenges of the knockout animals or in particularly permissive genetic backgrounds. Nevertheless, ideally investigations should be done in an in vivo context using animal models and primary cells. Further in-depth analyses may reveal a wider spectrum of lncRNA functions in the fine-tuning of GRNs controlling cell differentiation. This requires, among other approaches, further improvement of current methods for the identification of direct genomic targets of lncRNAs as well as for the purification and analysis of lncRNA/protein complexes, allowing their application to the numerous, low abundant lncRNA transcripts [61-63].

\section{Acknowledgments}

We thank Stefanie Dimmeler, Jesse Veenvliet, and Sandra Schmitz for comments on the manuscript. We apologize to those colleagues whose work is not mentioned in this review. This is entirely due to space restrictions and to the focus on organogenesis. The authors' work is funded by the Max Planck Society (B.G.H.) and the DFG (German Research Foundation) Excellence Cluster Cardio-Pulmonary System (Exc147-2) (P.G.).

\section{References}

1 Biben, C. et al. (2000) Cardiac septal and valvular dysmorphogenesis in mice heterozygous for mutations in the homeobox gene Nkx2-5. Circ. Res. 87, 888-895

2 Bruneau, B.G. et al. (2001) A murine model of Holt-Oram syndrome defines roles of the T-box transcription factor Tbx 5 in cardiogenesis and disease. Cell 106, 709-721

$3 \mathrm{Pu}$, W.T. et al. (2004) GATA4 is a dosage-sensitive regulator of cardiac morphogenesis. Dev. Biol. 275, 235-244

4 Djebali, S. et al. (2012) Landscape of transcription in human cells. Nature 489, 101-108

5 Salzman, J.et al. (2012) Circular RNAs are the predominant transcript isoform from hundreds of human genes in diverse cell types. PLoS ONE 7, e30733

6 Memczak, S. et al. (2013) Circular RNAs are a large class of animal RNAs with regulatory potency. Nature 495, 333-338

7 Guttman, M. et al. (2013) Ribosome profiling provides evidence that large noncoding RNAs do not encode proteins. Cell 154, 240-251

8 Ruiz-Orera, J. et al. (2014) Long non-coding RNAs as a source of new peptides. Elife 3, Published online September 16, 2014. http:// dx.doi.org/10.7554/eLife.03523

9 Dey, B.K. et al. (2014) The H19 long noncoding RNA gives rise to microRNAs miR-675-3p and miR-675-5p to promote skeletal muscle differentiation and regeneration. Genes Dev. Published online March 1, 2014. http://dx.doi.org/10.1101/gad.234419.113

10 Clark, M.B. et al. (2012) Genome-wide analysis of long noncoding RNA stability. Genome Res. 22, 885-898

11 Sigova, A.A. et al. (2013) Divergent transcription of long noncoding RNA/mRNA gene pairs in embryonic stem cells. Proc. Natl. Acad. Sci. U.S.A. 110, 2876-2881

12 Natoli, G. and Andrau, J-C. (2012) Noncoding transcription at enhancers: general principles and functional models. Annu. Rev. Genet. 46, 1-19

13 Marques, A.C. et al. (2013) Chromatin signatures at transcriptional start sites separate two equally populated yet distinct classes of intergenic long noncoding RNAs. Genome Biol. 14, Published online November 29, 2013. http://dx.doi.org/10.1186/gb-2013-14-11-r131 
14 Cabili, M.N. et al. (2011) Integrative annotation of human large intergenic noncoding RNAs reveals global properties and specific subclasses. Genes Dev. 25, 1915-1927

15 Werber, M. et al. (2014) The tissue-specific transcriptomic landscape of the mid-gestational mouse embryo. Development 141, 1-6

16 Ramos, A.D. et al. (2013) Integration of genome-wide approaches identifies lncRNAs of adult neural stem cells and their progeny in vivo. Cell Stem Cell 12, 616-628

$17 \mathrm{Ma}, \mathrm{W}$. et al. (2014) Fine-scale chromatin interaction maps reveal the cis-regulatory landscape of human lincRNA genes. Nat. Methods 12, $71-78$

18 Hnisz, D. et al. (2013) Super-enhancers in the control of cell identity and disease. Cell 155, 934-947

19 Zhao, J. et al. (2010) Genome-wide identification of polycombassociated RNAs by RIP-seq. Mol. Cell 40, 939-953

20 Khalil, A.M. et al. (2009) Many human large intergenic noncoding RNAs associate with chromatin-modifying complexes and affect gene expression. Proc. Natl. Acad. Sci. U.S.A. 106, 11667-11672

21 Cifuentes-Rojas, C. et al. (2014) Regulatory interactions between RNA and Polycomb Repressive Complex 2. Mol. Cell 55, 171-185

22 Morey, C. and Avner, P. (2011) The demoiselle ofX-inactivation: 50 years old and as trendy and mesmerising as ever. PLoS Genet. 7, Published online July, 2011. http://dx.doi.org/10.1371/journal.pgen.1002212

$23 \mathrm{Li}$, L. et al. (2013) Targeted disruption of Hotair leads to homeotic transformation and gene derepression. Cell Rep. 5, 3-12

24 Grote, P. et al. (2013) The tissue-specific lncRNA Fendrr is an essential regulator of heart and body wall development in the mouse. Dev. Cell 24, 206-214

25 Sauvageau, M. et al. (2013) Multiple knockout mouse models reveal lincRNAs are required for life and brain development. Elife 2, Published online December 31, 2013. http://dx.doi.org/10.7554/ eLife.01749

26 Klattenhoff, C.A. et al. (2013) Braveheart, a long noncoding RNA required for cardiovascular lineage commitment. Cell 152, 570-583

27 Scheuermann, J.C. and Boyer, L.A. (2013) Getting to the heart of the matter: long non-coding RNAs in cardiac development and disease. EMBO J. Published online June 11, 2013. http:dx.doi.org/10.1038/ emboj.2013.134

28 Wang, K.C. et al. (2011) A long noncoding RNA maintains active chromatin to coordinate homeotic gene expression. Nature 472, 120-124

29 Yang, Y.W. et al. (2014) Essential role of IncRNA binding for WDR5 maintenance of active chromatin and embryonic stem cell pluripotency. Elife 3, Published online February 2014. http:// dx.doi.org/10.7554/eLife.02046

30 Herriges, M.J. et al. (2014) Long noncoding RNAs are spatially correlated with transcription factors and regulate lung development. Genes Dev. 28, 1363-1379

31 Di Ruscio, A. et al. (2013) DNMT1-interacting RNAs block gene-specific DNA methylation. Nature 503, 371-376

32 Chalei, V. et al. (2014) The long non-coding RNA Dali is an epigenetic regulator of neural differentiation. Elife 3, Published online November 21, 2014. http://dx.doi.org/10.7554/eLife.04530

33 Tsai, M-C. et al. (2010) Long noncoding RNA as modular scaffold of histone modification complexes. Science 329, 689-693

34 Kanduri, C. (2011) Kenq1ot1: a chromatin regulatory RNA. Semin. Cell Dev. Biol. 22, 343-350

35 Feng, J. et al. (2006) The Evf-2 noncoding RNA is transcribed from the Dlx-5/6 ultraconserved region and functions as a Dlx-2 transcriptional coactivator. Genes Dev. 20, 1470-1484

36 Berghoff, E.G. et al. (2013) Evf2 (Dlx6as) lncRNA regulates ultraconserved enhancer methylation and the differential transcriptional control of adjacent genes. Development 140, 4407-4416

37 Bond, A.M. et al. (2009) Balanced gene regulation by an embryonic brain ncRNA is critical for adult hippocampal GABA circuitry. Nat. Neurosci. 12, 1020-1027

38 Uhde, C.W. et al. (2010) Rmst is a novel marker for the mouse ventral mesencephalic floor plate and the anterior dorsal midline cells. PLoS ONE 5, Published online January 8, 2010. http://dx.doi.org/10.1371/ journal.pone.0008641

$39 \mathrm{Ng}$, S-Y. et al. (2012) Human long non-coding RNAs promote pluripotency and neuronal differentiation by association with chromatin modifiers and transcription factors. EMBO J. 31, 522-533
$40 \mathrm{Ng}$, S-Y. et al. (2013) The long noncoding RNA RMST interacts with SOX2 to regulate neurogenesis. Mol. Cell 51, 349-359

41 Rapicavoli, N.A. et al. (2011) The long noncoding RNA Six3OS acts in trans to regulate retinal development by modulating Six3 activity. Neural Dev. 6., Published online September 21, 2011. http://dx.doi.org/ 10.1186/1749-8104-6-32

42 Lin, N. et al. (2014) An evolutionarily conserved long noncoding RNA TUNA controls pluripotency and neural lineage commitment. Mol. Cell Published online March 20, 2014. http://dx.doi.org/10.1016/ j.molcel.2014.01.021

43 Ding, L. et al. (2009) A genome-scale RNAi screen for Oct4 modulators defines a role of the Paf1 complex for embryonic stem cell identity. Cell Stem Cell 4, 403-415

44 Chia, N-Y. et al. (2010) A genome-wide RNAi screen reveals determinants of human embryonic stem cell identity. Nature 468, $316-320$

45 Ulitsky, I. et al. (2011) Conserved function of lincRNAs in vertebrate embryonic development despite rapid sequence evolution. Cell 147, $1537-1550$

46 Rocha, P.P. et al. (2010) Med12 is essential for early mouse development and for canonical Wnt and Wnt/PCP signaling. Development 137, 2723-2731

47 Lai, F. et al. (2013) Activating RNAs associate with Mediator to enhance chromatin architecture and transcription. Nature 494, $497-501$

48 Ounzain, S. et al. (2014) Functional importance of cardiac enhancerassociated noncoding RNAs in heart development and disease. J. Mol. Cell. Cardiol. 76C, 55-70

49 Kretz, M. et al. (2012) Control of somatic tissue differentiation by the long non-coding RNA TINCR. Nature 493, 231-235

50 Mattick, J.S. (2001) Non-coding RNAs: the architects of eukaryotic complexity. EMBO Rep. 2, 986-991

51 Mattick, J.S. (2011) The central role of RNA in human development and cognition. FEBS Lett. 585, 1600-1616

52 Mercer, T.R. et al. (2008) Specific expression of long noncoding RNAs in the mouse brain. Proc. Natl. Acad. Sci. U.S.A. 105, 716-721

53 Batista, P.J. and Chang, H.Y. (2013) Long noncoding RNAs: cellular address codes in development and disease. Cell 152, 1298-1307

54 Bassett, A.R. et al. (2014) Considerations when investigating lncRNA function in vivo. Elife 3, Published online August 14, 2014. http:// dx.doi.org/10.7554/eLife.03058

55 Tripathi, V. et al. (2010) The nuclear-retained noncoding RNA MALAT1 regulates alternative splicing by modulating SR splicing factor phosphorylation. Mol. Cell 39, 925-938

56 Tripathi, V. et al. (2013) Long noncoding RNA MALAT1 controls cell cycle progression by regulating the expression of oncogenic transcription factor B-MYB. PLoS Genet. 9, Published online March 21, 2013. http://dx.doi.org/10.1371/journal.pgen.1003368

57 Gutschner, T. et al. (2013) The noncoding RNA MALAT1 is a critical regulator of the metastasis phenotype of lung cancer cells. Cancer Res. Published online February 1, 2013. http://dx.doi.org/10.1158/00085472.CAN-12-2850

58 Huarte, M. et al. (2010) A large intergenic noncoding RNA induced by p53 mediates global gene repression in the p53 response. Cell 142, 409-419

59 Dimitrova, N. et al. (2014) LincRNA-p21 activates p21 in cis to promote polycomb target gene expression and to enforce the G1/S checkpoint. Mol. Cell 54, 777-790

60 Kok, F.O. et al. (2014) Reverse genetic screening reveals poor correlation between Morpholino-induced and mutant phenotypes in zebrafish. Dev. Cell 32, 97-108

61 Chu, C. et al. (2011) Genomic maps of long noncoding RNA occupancy reveal principles of RNA-chromatin interactions. Mol. Cell 44, 667-678

62 Simon, M.D. et al. (2011) The genomic binding sites of a noncoding RNA. Proc. Natl. Acad. Sci. U.S.A. 108, 20497-20502

63 Engreitz, J.M. et al. (2013) The Xist lncRNA exploits three-dimensional genome architecture to spread across the X chromosome. Science 341. Published online July 4, 2013. http://dx.doi.org/10.1126/science. 1237973

64 Vance, K.W. et al. (2014) The long non-coding RNA Paupar regulates the expression of both local and distal genes. EMBO J. 33, 296-311 